\title{
Correcting quantum errors with the Zeno effect
}

\author{
Noam Erez, ${ }^{1,3}$ Yakir Aharonov, ${ }^{1,2}$ Benni Reznik, ${ }^{1}$ and Lev Vaidman ${ }^{1}$ \\ ${ }^{1}$ School of Physics and Astronomy, Tel Aviv University, Tel Aviv 69978, Israel \\ ${ }^{2}$ Department of Physics, University of South Carolina, Columbia, South Carolina 29208, USA \\ ${ }^{3}$ Institute for Quantum Studies and Department of Physics, Texas A\&M University, College Station, Texas 77843-4242, USA
}

(Received 4 November 2003; published 15 June 2004)

\begin{abstract}
In order to reduce errors, error correction codes need to be implemented fast. They can correct the errors corresponding to the first few orders in the Taylor expansion of the time evolution operator corresponding to the Hamiltonian of the interaction with the environment. If implemented fast enough, the zeroth order error predominates and the dominant effect is of error prevention by measurement (Zeno effect) rather than correction. In this "Zeno regime," codes with less redundancy are sufficient for protection. We describe such a simple scheme, which uses two "noiseless" qubits to protect a large number, $n$, of information qubits from noise from the environment. The "noisless qubits" can be realized by treating them as logical qubits to be encoded by one of the previously introduced encoding schemes.
\end{abstract}

DOI: 10.1103/PhysRevA.69.062315

\section{INTRODUCTION}

Quantum error correction schemes based on encoding, consist of the following steps: (1) encoding $n$ logical qubits into $m>n$ physical ones, (2) introduction of errors, (3) syndrome measurement-projecting the state onto one of a number of subspaces corresponding to a discrete set of errors, (4) error correction [1]. For a review on various error correction codes see Ref. [3].

The error correction capabilities of the various codes are usually described in terms of the discrete set of errors in step (3): a code is said to correct a discrete set of errors with certainty. This makes the treatment very similar to the theory of algebraic codes of classical information theory.

In most of the error correction codes that have been proposed, the discrete set of errors to be corrected consists of Pauli operators acting on only a few qubits. Why is this error set interesting? If one assumes that the different qubits are located at well separated physical locations (as in an ion-trap realization of a quantum computer), then it is reasonable to assume that the environmentally induced errors for the different qubits are independent. If the correction is implemented fast enough, then the dominant contribution will come from the first few orders in the Taylor expansion of the interaction with the environment, and these are spanned by the discrete set.

It is important to note, that one of the syndromes corresponds to "no-error," and that this corresponds to the zeroth order error. Therefore, implementing steps (1)-(3) often enough will also have the effect of reducing the error [8]. In other words, measuring the syndrome often enough prevents errors. This is the quantum Zeno effect [4] (QZE) (for the syndrome degree of freedom!): repeatedly making a projective measurement can freeze the dynamics. For a discussion of the implementability of the QZE, as well as the inverse effect, see Ref. [5] A number of quantum codes utilizing the error prevention that occurs in the Zeno limit have been proposed [6-8].

In the "Zeno regime," the "first order" error correction codes are overly redundant. It has been shown in Ref. [8] that in that regime, the 5-qubit code [2] can be replaced by a
PACS number(s): 03.67.Pp, 03.65.Xp, 03.65.Yz, 03.67.Lx

2-qubit encoding per logical qubit (for an even number of logical qubits). In other words, error correction codes can protect $n$ logical qubits which are encoded in $5 n$ physical qubits[2], and previous error prevention codes (Zeno) can achieve this for encoding in $2 n$ physical qubits.

In the next section we describe a "Zeno" error correction code which encodes $n$ logical qubits in $n+4$ physical qubits. This consists of two encoding steps. The first step consists of encoding into $n+2$ qubits for a code that protects against single qubit errors that can occur in only a definite set of $n$ qubits. The second step is to encode the two unprotected qubits with the 4-qubit code of Ref. [8].

The typical decoherence time scales, as well as rates of computation, for various systems utilized in various proposed quantum computers can be found in Ref. [9]. Reference [10] suggests that a particular type of noise (" $1 / f$ noise") is the dominant one for many solid state systems and analyzes the effect of dynamical decoupling on it. Dynamical decoupling [13] (DD) schemes of EC, utilize repeated perturbation of the system by a strong field which rotates the state in Hilbert space, to average out noise. While this is also described sometimes as a Zeno effect, it differs from our scheme in a number of ways. DD has the advantage over many other schemes, that it can effectively deal with errors effecting many spins at once, but on the other hand requires assumptions about symmetry properties of the errors. The rate of "correction" pulses required in this scheme is governed by the dominant frequencies of the noise, no matter how weak it is. On the other hand, in the proposed scheme, it is the magnitude of the noise (the magnitude of the matrix elements of the noise operator) which is important. A method called adiabatic quantum computation [11] has been shown to be applicable to various combinatorial search problems. The algorithm consists of smoothly varying a Hamiltonian from one that has an easily prepared ground state, to one whose ground state encodes the solution. It was shown[12] that in the adiabatic limit, not only is the algorithm carried out under noisless conditions, but in fact is also immune to decoherence. The required rate is determined by the gap between the instantaneous ground state and the rest of the spectrum, and it is not known how to determine it in general. 
Recently, protocols combining several error correction techniques have been proposed[14], and a unification of dynamical decoupling and the QZE has been suggested[15] (for a time independent noise Hamiltonian).

\section{A ZENO ERROR PREVENTION CODE}

In close analogy to the linear codes of classical information theory, a quantum code which encodes $n$ logical qubits into $m=n+r$ physical qubits is defined to be a unitary linear mapping $C: \mathcal{H}_{\text {logical }} \mapsto \mathcal{H}_{\text {physical }}$ where $\operatorname{dim}\left(\mathcal{H}_{\text {physical }}\right)=2^{m}$ and $\operatorname{dim}\left(\mathcal{H}_{\text {physical }}\right)=2^{n}$. This must be implemented by a unitary acting on a Hilbert space which includes $\mathcal{H}_{\text {logical }}$ and $\mathcal{H}_{\text {physical }}$ as subspaces. Nothing essential is lost if we assume that this large Hilbert space is simply $\mathcal{H}_{\text {physical }}$. This just means that the logical qubits are initially stored in a $2^{m}$ dimensional subspace of $\mathcal{H}_{\text {physical }}$. Then $\mathcal{H}_{\text {physical }}$ is isomorphic to the product $\mathcal{H}_{\text {system }} \otimes \mathcal{H}_{\text {ancilla }}$ where $\mathcal{H}_{\text {system }}$ is isomorphic to $\mathcal{H}_{\text {logical }}$ and $\mathcal{H}_{\text {ancilla }}$ is a dimensional space we will call the "ancilla." Now to fix the identity of the subspace $\mathcal{H}_{\text {logical }}$, we have to choose a one-dimensional subspace of $\mathcal{H}_{\text {ancilla }}$, denoted by $\operatorname{Span}\left\{|i n\rangle_{\text {ancilla }}\right\}$ (where "in" stands for initial state), so $\mathcal{H}_{\text {logical }}=\mathcal{H}_{\text {sys }} \otimes|i n\rangle_{\text {anc }}$. To summarize, we rewrite the encoding operation: "state of $m$ logical qubits is mapped into state of $n$ physical qubits," as " $|\psi\rangle_{\text {sys }} \otimes|i n\rangle_{a n c}$ is mapped to encoded state of same space." Formally then, the encoding operation corresponds to an operator $C$,

$$
C: \mathcal{H}_{\text {sys }} \otimes \mathcal{H}_{\text {anc }} \mapsto \mathcal{H}_{\text {sys }} \otimes \mathcal{H}_{\text {anc }} .
$$

After this encoding step, some interaction with the environment introduces noise. ${ }^{1}$ The most general Hamiltonian describing an environment (a system with state Hilbert space, $\mathcal{H}_{\text {env }}$ ) interacting independently with each system qubit is

$$
H_{\text {noise }}=-\mathcal{E} \sum_{\substack{i=1, \ldots, n \\ b=0, \ldots, 3}} \sigma_{b}^{s_{i}} \otimes A_{e n v}^{i, b},
$$

where $\left\{\sigma_{b}^{s_{i}}\right\}$ designates the set $\left\{1, \sigma_{x}, \sigma_{y}, \sigma_{z}\right\}$ acting on the $i$ th system qubit. The time evolution for short times, $t=\varepsilon \hbar / \mathcal{E}$, is then

$$
N(\varepsilon) \equiv U_{\text {noise }}(\varepsilon \hbar / \mathcal{E})=1+i \varepsilon \sum_{i, b} \sigma_{b}^{s_{i}} \otimes A_{e n v}^{i, b}+O\left(\varepsilon^{2}\right) .
$$

We claim that it suffices to use a 2-qubit ancilla (i.e., $\left.\operatorname{dim} \mathcal{H}_{\text {anc }}=4\right)$ to protect an unknown state in $\mathcal{H}_{\text {sys }}\left(\operatorname{dim} \mathcal{H}_{\text {sys }}\right.$ $=2^{n}$ ) from the first order terms in the expansion of the noise operator, Eq. (3). Namely, for a certain choice of the initial state of the ancilla, $|i n\rangle_{\text {anc }}$, and encoding operator, ${ }^{2} C$, which will be specified below

\footnotetext{
${ }^{1}$ Note that in a more realistic model, we would have to consider noise during all the other steps as well, which would require a fault tolerant approach.

${ }^{2}$ We make here two implicit assumptions about the environment. First, we assume (in common with most works on error correction) that it is initially in a product state with our ancilla and system. Second, we assume its initial state to be pure. The latter assumption is not really needed, but was made in order to simplify the notation.
}

$$
C N C|i n\rangle|\psi\rangle|0\rangle_{e n v}=|i n\rangle_{\text {anc }}|\psi\rangle_{s}|\phi\rangle_{e n v}+\varepsilon|\perp\rangle+O\left(\varepsilon^{2}\right)\left|\Psi^{\prime}\right\rangle,
$$

where $|\perp\rangle \in|i n\rangle_{\text {anc }}^{\perp} \otimes \mathcal{H}_{\text {sys }} \otimes \mathcal{H}_{\text {env }}$ and $\left|\Psi^{\prime}\right\rangle$ is some arbitrary state of the system, ancilla and the environment. While it is to be expected that $|\perp\rangle$ should be orthogonal to the initial overall state, it should be emphasized that it is orthogonal to the initial state of the ancilla. This means that a projective measurement on the ancilla alone will find it in the initial state $|i n\rangle$ with probability $1-O\left(\varepsilon^{2}\right)$, and in this case the system will be in its (unknown) initial state, $|\psi\rangle$ (also with probability $\left.1-O\left(\varepsilon^{2}\right)\right)$. The $O\left(\varepsilon^{2}\right)$ probability not to find the system in state $|\psi\rangle$ even for a favorable outcome of the measurement of the ancilla, corresponds to the $O\left(\varepsilon^{2}\right)$ terms in Eq. (3), i.e., to higher order errors ( $n$ qubit errors, $n>1$ ).

Let us show this for a 2-qubit ancilla, assuming the noise acts only on the other $n$ qubits. The logical qubits reside originally in $|\psi\rangle \in \mathcal{H}_{s y s}\left(\operatorname{dim} \mathcal{H}_{s y s}=2^{n}\right)$. Let us choose some arbitrary basis $\{|a\rangle\}_{a=0}^{3}$ for $\mathcal{H}_{\text {anc }}$, and choose $\mid$ in $\rangle_{a n c}$ $=\frac{1}{2} \sum_{a=0}^{3}|a\rangle$, and define our encoding operator, $C$, to be

$$
C=\sum_{a=0}^{3}(|a\rangle\langle a|) \otimes \prod_{i=1}^{n} \sigma_{a}^{i},
$$

where $\sigma_{a}^{i}$ acts on the $i$ th qubit in $\mathcal{H}_{\text {sys. }}$. Then the encrypted $n+2$ qubit state is

$$
C|i n\rangle_{\text {anc }}|\psi\rangle_{s y s}=\frac{1}{2} \sum_{a=0}^{3}|a\rangle\left(\otimes_{i=1}^{n} \sigma_{a}^{i}\right)|\psi\rangle .
$$

Then for $b \neq 0$,

$$
\begin{aligned}
C \sigma_{b}^{j} C & =\sum_{a}|a\rangle\langle a|\left(\otimes_{i} \sigma_{a}^{i}\right) \sigma_{b}^{j}\left(\otimes_{i^{\prime}} \sigma_{a}^{i^{\prime}}\right) \\
& =\sum_{a}|a\rangle\langle a| \sigma_{a}^{j} \sigma_{b}^{j} \sigma_{a}^{j}=\left(\sum_{a}|a\rangle\langle a| c_{a}^{(b)}\right) \sigma_{b}^{j},
\end{aligned}
$$

where $c_{a}^{(b)}=\left\{\begin{array}{ll}+1, & a=0, b \\ 1 & \text { otherwise. }\end{array}\right.$.

For $b=0$, we have the trivial result $c_{a}^{(0)}=1$ for all $a$. For all $b$ then

$$
C \sigma_{b}^{j} C|i n\rangle|\psi\rangle=\left(\frac{1}{2} \sum c_{a}^{(b)}|a\rangle\right) \sigma_{b}^{j}|\psi\rangle \equiv|\widetilde{b}\rangle \sigma_{b}^{j}|\psi\rangle,
$$

the $\{|\widetilde{b}\rangle\}_{b=0, \ldots, 3}$ form an orthonormal basis, and $|\widetilde{0}\rangle=|i n\rangle{ }^{3}$

Finally, we can write the effect of encoding on the environmentally induced errors (we assume, as before, that initially the system is in a product state with the environment, and that the latter is in a pure state, $|0\rangle_{\text {env }}$ ):

If the scheme works for any pure state, by linearity it will work for any mixture as well. The first assumption is more subtle.

${ }^{3}$ It is not hard to see that if the original basis $\{|a\rangle\}_{a=0, \ldots, 3}$ is chosen as the simultaneous eigenfunctions of $\sigma_{z} \otimes 1,1 \otimes \sigma_{z}$, the $\{|\tilde{b}\rangle\}_{b=0, \ldots, 3}$ basis consists of the simultaneous eigenfunctions of $\sigma_{x} \otimes 1,1 \otimes \sigma_{x}$. 


$$
\begin{aligned}
& C N C \mid \text { in }\rangle_{\text {anc }}|\psi\rangle_{\text {sys }}|0\rangle_{\text {env }} \\
& =|\widetilde{0}\rangle_{\text {anc }}|\psi\rangle_{\text {sys }}|\phi\rangle_{\text {env }}+i \varepsilon \sum_{\substack{b \neq 0 \\
j=1, \ldots, m}}|\tilde{b}\rangle_{e n v} \sigma_{b}^{j}|\psi\rangle_{s} A^{j, b}|0\rangle_{\text {env }} \\
& \quad+O\left(\varepsilon^{2}\right)\left|\psi^{\prime}\right\rangle=|i n\rangle_{\text {anc }}|\psi\rangle_{s}|\phi\rangle_{\text {env }}+\varepsilon|\perp\rangle+O\left(\varepsilon^{2}\right)\left|\psi^{\prime}\right\rangle .
\end{aligned}
$$

So far we have, somewhat artificially, considered the case where we have at our disposal 2 "privileged" qubits which are exempt from noise (the 2 qubits we had singled out as our ancilla). To remedy this, we note that these ancillary qubits can be each encoded in 5 "ordinary" physical qubits subject to the same noise as the others, using the famous 5-qubit code [2]. Alternatively, we can encode the two special qubits in 4 physical qubits using the scheme of Ref. [8] to get an "all Zeno" code.

\section{TWO INTERPRETATIONS}

In this section we will mainly restrict the discussion to the case of one logical qubit $(n=1)$ for the sake of simplicity. The generalization to the case of arbitrary $n$ is straightforward, and will be discussed very briefly.

\section{A. Heisenberg representation}

In the previous sections, we have concentrated on the Schrödinger representation to make the treatment more easily comparable to the usual error correction schemes. However, a few authors have also looked at error correction in the Heisenberg representation, see for example [16]. Our original derivation was in the latter representation, and is perhaps somewhat more natural. In the Heisenberg representation, the encoding and decoding operators, which act before and after the "noise operator", respectively, are seen as acting on the latter operator. The choice of initial state of the ancilla, and the projection onto the syndrome subspaces (also defined by the state of the ancilla in our scheme) are seen as pre- and post-selection steps. The desired effect of the encoding, decoding and postselection should be that when the postselection of the desired subspace succeeds, the effective noise operator becomes trivial as far as the system is concerned.

To be more concrete, let us write $N$ for the noise operator which acts on our system and the environment:

$$
N: \mathcal{H}_{\text {sys }} \otimes \mathcal{H}_{\text {env }} \mapsto \mathcal{H}_{\text {sys }} \otimes \mathcal{H}_{\text {env }},
$$

$U_{e n c}, U_{d e c}$ for the encoding and decoding operators (respectively) acting on the system and ancilla:

$$
U_{\text {enc,dec }}: \mathcal{H}_{\text {sys }} \otimes \mathcal{H}_{\text {anc }} \mapsto \mathcal{H}_{\text {sys }} \otimes \mathcal{H}_{\text {anc }}
$$

and $\mid$ in $\rangle$ and $\mid$ out $\rangle$ for the preselected (respectively, postselected) states of the ancilla, then

$$
{ }_{\text {anc }}\left\langle\text { out }\left|U_{\text {dec }} N U_{\text {enc }}\right| i n\right\rangle_{\text {anc }} \propto 1_{\text {sys }} .
$$

Before we write the explicit form of these objects in our scheme, let us motivate it with a simple example that works for a single qubit "system" a single qubit ancilla and a noise operator of the special form $N=O_{0}^{e n v}+O_{1}^{e n v} \sigma_{y}^{s y s}+O_{2}^{e n v} \sigma_{z}^{s y s}$ (i.e. not involving $\sigma_{x}$ ). For the usual definition of the CNOT (conditional flip) operator:

$$
\begin{gathered}
C_{x}=(|0\rangle\langle 0|)_{\text {ancilla }}+(|1\rangle\langle 1|)_{\text {ancilla }} \sigma_{x}^{\text {sys }}, \\
\left(|0\rangle \equiv\left|s_{z}=+\hbar / 2\right\rangle, \quad|1\rangle \equiv\left|s_{z}=-\hbar / 2\right\rangle\right),
\end{gathered}
$$

we have the following property:

$$
C_{x} \sigma_{y}^{s y s} C_{x}=\sigma_{y}^{s y s} \sigma_{z}^{a n c}, \quad C_{x} \sigma_{z}^{s y s} C_{x}=\sigma_{z}^{s y s} \sigma_{z}^{a n c}
$$

and so

$$
\operatorname{anc}_{x}\left\langle\uparrow_{x}|C N C| \uparrow_{x}\right\rangle_{\text {anc }}=O_{0}^{\text {env }},
$$

which is Eq. (10) with $U_{e n c}=U_{d e c}=C$ and $\mid$ in $\rangle_{\text {ancilla }}$ $=\mid$ out $\rangle_{\text {ancilla }}=\left|\uparrow_{x}\right\rangle$.

In order to generalize this treatment to treat general errors (for a one qubit system), we will need two "conditional-flip" operators, where the "flip" denotes a Pauli operator: $C_{a}^{1,2}$ $=|0\rangle_{1}\left\langle 0\left|\otimes 1_{2}+\right| 1\right\rangle_{1}\langle 1| \otimes \sigma_{a}^{2}(a=x, y, z)$ which acts on $\mathcal{H}_{1} \otimes \mathcal{H}_{2}$. Writing $\mathcal{H}_{\text {anc }}=\mathcal{H}_{1} \otimes \mathcal{H}_{2}, \mathcal{H}_{\text {sys }}=\mathcal{H}_{3}$, and $C=C_{y}^{1,3} C_{z}^{2,3}$ (regular operator product), then Eq. (11) generalizes to

$$
\begin{aligned}
& C\left(1_{a n c} \otimes \sigma_{x}\right) C^{\dagger}=\left(\sigma_{z} \otimes \sigma_{z}\right)^{a n c} \otimes\left(\sigma_{x}\right)^{s y s}, \\
& C\left(1_{a n c} \otimes \sigma_{y}\right) C^{\dagger}=\left(1 \otimes \sigma_{z}\right)^{a n c} \otimes\left(\sigma_{y}\right)^{s y s}, \\
& C\left(1_{a n c} \otimes \sigma_{z}\right) C^{\dagger}=\left(\sigma_{z} \otimes 1\right)^{a n c} \otimes\left(\sigma_{z}\right)^{s y s},
\end{aligned}
$$

which follow from

$$
\begin{aligned}
C_{a}^{1,2} \sigma_{b}^{2} C_{a}^{1,2} & =(|0\rangle\langle 0|)_{1} \sigma_{b}^{2}+(|1\rangle\langle 1|)_{1}\left(\sigma_{a} \sigma_{b} \sigma_{a}\right)_{2} \\
& =\left[|0\rangle\langle 0|+| 1\rangle\langle 1|(-1)^{\delta_{a b}+1}\right]_{1} \sigma_{b}^{2} \\
& =\left\{\begin{array}{c}
1 \otimes \sigma_{b} a=b, \\
\sigma_{z} \otimes \sigma_{b} a \neq b .
\end{array}\right.
\end{aligned}
$$

Note also that

$$
\begin{aligned}
C= & (|0,0\rangle\langle 0,0|)_{a n c} 1_{\text {sys }}+(|0,1\rangle\langle 0,1|)_{a n c} \sigma_{z}^{\text {sys }} \\
& +(|1,0\rangle\langle 1,0|)_{a n c} \sigma_{y}^{\text {sys }}+i(|1,1\rangle\langle 1,1|)_{a n c} \sigma_{x}^{\text {sys }},
\end{aligned}
$$

which is almost our definition (5) for $n=1$.

Let us introduce for these expressions the more compact notation:

$$
C \sigma_{a}^{s y s} C^{\dagger}=\Sigma_{a}^{a n c} \sigma_{a}^{s y s}
$$

and add $\Sigma_{0}=1$.

With the definition, $\mid$ in $\rangle=\left|\uparrow_{x}, \uparrow_{x}\right\rangle$, and the fact that anc $\left\langle\text { in }\left|\Sigma_{a}\right| i n\right\rangle_{a n c}=\delta_{a 0}$, we finally have

$$
\begin{aligned}
& { }_{a n c}\left\langle\text { in }\left|C U C^{\dagger}\right| \text { in }\right\rangle_{a n c} \\
& \quad \simeq{ }_{a n c}\left\langle i n\left|\exp \left(i \varepsilon \sum_{i, a} H_{i, a}^{e n v} \sum_{a}^{a n c, s_{i}} \sigma_{a}^{s_{i}}\right)\right| i n\right\rangle_{a n c} \\
& \quad \simeq \exp \left(i \varepsilon \sum H_{i, a}\left\langle i n\left|\Sigma_{a}^{a n c, s_{i}}\right| i n\right\rangle \sigma_{a}^{s_{i}}\right) \\
& \quad=\exp \left(i \varepsilon \sum_{i} H_{i, 0}^{e n v}\right) \otimes 1^{\text {sys }} .
\end{aligned}
$$


This last result can be interpreted as follows: for an ancilla initially in state $|i n\rangle$, and postselected to be in the same state, the operator $U$ is effectively reduced to a harmless one acting on the environment alone. In this form, it is very reminiscent of dynamical error correction. Nevertheless, this approach has the advantage, that unlike dynamical error correction, it does not require that the noise be slowly varying, only that it be "small."

\section{B. Error prevention as teleportation in time}

Our scheme can also be written in yet another way, related to measurement theory, if we look at the controlled-not operation as a measurement of the state of the system qubit.

It was shown in [19] that "crossed nonlocal measurements" performed on two separated qubits

$$
\left[\sigma_{1 x}\left(t_{1}\right)-\sigma_{2 x}\left(t_{1}+\epsilon\right)\right] \bmod 4, \quad\left[\sigma_{1 y}\left(t_{1}+\epsilon\right)-\sigma_{2 y}\left(t_{1}\right)\right] \bmod 4
$$

yield two-way teleportation or "swapping" of the states of the qubits. The four possible outcomes of these measurements define the correction which have to be performed on the qubits to complete the teleportation. The particular results

$$
\left[\sigma_{1 x}\left(t_{1}\right)-\sigma_{2 x}\left(t_{1}+\epsilon\right)\right] \bmod 4=\left[\sigma_{1 y}\left(t_{1}+\epsilon\right)-\sigma_{2 y}\left(t_{1}\right)\right] \bmod 4=0
$$

correspond to immediate teleportation without need for corrections. In this teleportation procedure, the times of the interaction with the second qubit can be changed provided that the order remains the same

$$
\left[\sigma_{1 x}\left(t_{1}\right)-\sigma_{2 x}\left(t_{2}+\epsilon\right)\right] \bmod 4, \quad\left[\sigma_{1 y}\left(t_{1}+\epsilon\right)-\sigma_{2 y}\left(t_{2}\right)\right] \bmod 4 .
$$

In particular, we can arrange that $t_{2}>t_{1}+\epsilon$. The "identity" of the second particle is not important, the procedure teleports the state of the qubit to any particle with which the interactions are performed. Thus, we can make the interactions at times $t_{2}$ and $t_{2}+\epsilon$ with the particle which had the first qubit. In this case we teleport the quantum state of a particle to the particle itself, but at a later time: teleportation in time!

In fact, teleportation in time (as well as teleportation to another particle at time-like interval) is much easier to perform than teleportation to a space-like interval. We have to perform the following two-time measurements [17]

$$
\begin{aligned}
& {\left[\sigma_{x}\left(t_{1}\right)-\sigma_{x}\left(t_{2}+\epsilon\right)\right] \bmod 4,} \\
& {\left[\sigma_{y}\left(t_{1}+\epsilon\right)-\sigma_{y}\left(t_{2}\right)\right] \bmod 4,}
\end{aligned}
$$

where $t_{1}<t_{2}<t_{1}^{\prime}<t_{2}^{\prime}$. These measurements are much easier to perform than measurements of nonlocal variables required for the two-way teleportation. There is no need to have entangled particles in the measuring device. A single qubit replaces the entangled pair. The coupling to the qubit is the same as the coupling to the entangled qubits of the pair and, in fact, it is just CNOT in the appropriate basis, exactly the same interaction which was used in the procedure described in the previous section.
The measurements (17) and, in general, the measurements (20) might have four possible outcomes. However, if our system was not disturbed between $t_{1}$ and $t_{2}^{\prime}$ (except for measurements (20)), only a single outcome is possible:

$$
\left[\sigma_{x}\left(t_{1}\right)-\sigma_{x}\left(t_{2}+\epsilon\right)\right] \bmod 4=\left[\sigma_{y}\left(t_{1}+\epsilon\right)-\sigma_{y}\left(t_{2}\right)\right] \bmod 4=0 .
$$

This is the outcome which corresponds to the teleportation without corrections. Indeed, the measurements (20) are also verification measurements of the particular type of a twotime state [18]

$$
\Psi_{t_{1}, t_{2}}=\frac{1}{\sqrt{2}}\left(\left\langle\left.\uparrow\right|_{t_{1}} \mid \uparrow\right\rangle_{t_{2}}+\left\langle\downarrow\left|t_{1}\right| \downarrow\right\rangle_{t_{2}},\right.
$$

which is generated by vanishing Hamiltonian at the time period $\left[t_{1}, t_{2}\right]$. If, during this period there will be a small disturbance then the measurement, due to Zeno effect will, with high probability, still have the outcome (21) and it also will nullify the action of the disturbance, i.e., prevent errors during this time.

For discussion of the general case of protection $N$ qubits we have to look more closely on the process of measurement of the two time-variables (20). These measurements require two qubits prepared in a particular state before time $t_{1}$ which undergo two CNOT (conditional flip) interactions. One qubit in the $\sigma_{x}$ basis at times $t_{1}$ and $t_{2}+\epsilon$ and another, in the $\sigma_{y}$ basis at times $t_{1}+\epsilon$ and $t_{2}$. The measurements, after the interaction with the system which verify that the test qubits have not changed their state, complete the measurement. If the system was not disturbed, then the coupling of the test qubits with the system does not prevent the test qubits to verify the absence of disturbing of another qubit using coupling corresponding to two-time measurements performed on another qubit

$$
\begin{aligned}
& {\left[\sigma_{x}^{\prime}\left(t_{1}-\delta\right)-\sigma_{x}\left(t_{2}+\epsilon+\delta\right)\right] \bmod 4,} \\
& {\left[\sigma_{y}\left(t_{1}+\epsilon-\delta\right)-\sigma_{y}\left(t_{2}+\delta\right)\right] \bmod 4 .}
\end{aligned}
$$

If both systems are under small disturbance (such that probability of flipping of both qubits is negligible) then the procedure: preparation of the test qubits coupling of the test qubits with the two systems, and final verification that the test qubits have not changed their state will lead, through Zeno effect, to prevention of errors in the two systems. The general case is treated as before.

It is hard to make a concrete proposal for practical application of our method since present technology is still far from operating large numbers of qubits coherently. We might try to speculate that the method can be implemented in the future in a quantum computer or quantum memory of a cryptographic device operating with $n$ qubits (ions in a trap?) subject to moderate decoherence. Then, between the times of gate operations we send two particles of another kind which 
have a smaller coupling with the environment (polarized photons?) to interact successively with the qubits as we described above. Since other proposals for quantum error correction require a larger number of qubits, our proposal can be one of the first to be implemented in practice.

\section{ACKNOWLEDGMENTS}

We would like to acknowledge the support of the Basic Research Foundation of the Israeli Academy of Sciences and Humanities. Y.A. acknowledges the support of the National Science Foundation.
[1] P. Shor, Phys. Rev. A 52, R2493 (1995).

[2] C. H. Bennett, D. P. DiVincenzo, J. A. Smolin, and W. K. Wooters, Phys. Rev. A 54, 3824 (1996); R. Laflamme, C. Miquel, J. P. Paz, and W. H. Zurek, Phys. Rev. Lett. 77, 198 (1996).

[3] E. Knill, R. Laflamme, A. Ashikmin, H. Barnum, L. Viola, and W. H. Zurek e-print quant-ph/0207170.

[4] B. Misra and E. C. G. Sudarshan, J. Math. Phys. 18, 756 (1977).

[5] A. G. Kofman and G. Kurizki, Phys. Rev. Lett. 87, 270405 (2001).

[6] W. Zurek, Phys. Rev. Lett. 53, 391 (1984).

[7] A. Barenco, A. Berthiaume, D. Deutsch, A. Eckert, R. Josza and C. Macchiavello, SIAM J. Comput. 26, 1541 (1997).

[8] L. Vaidman, L. Goldenberg, and S. Wiesner, Phys. Rev. A 54, R1745 (1996).

[9] T. D. Ladd, D. Maryenko and Y. Yamamoto, e-print quant-ph/ 0309164.
[10] L. Faoro and L. Viola, e-print quant-ph/0312159.

[11] E. Farhi, J. Goldstone, S. Gutman, and M. Sipser, e-print quant-ph/0001106.

[12] A. Childs, E. Farhi, and J. Preskill, Phys. Rev. A 65, 012322 (2002).

[13] L. Viola and S. Lloyd, Phys. Rev. A 58, 2733 (1998).

[14] M. S. Byrd and D. A. Lidar, Phys. Rev. Lett. 89, 047901 (2002); J. Mod. Opt. 50, 1285 (2003); K. Khodjasteh and D. A. Lidar, Phys. Rev. A 68, 022322 (2003).

[15] P. Facchi, D. A. Lidar and S. Pascazio, e-print quant-ph/ 0303132.

[16] D. Gottesman, e-print quant-ph/9807006.

[17] Y. Aharonov, D. Albert, and S. D'Amato, Phys. Rev. Lett. 56, 2427 (1986).

[18] L. Vaidman, Ph.D. thesis, 1987.

[19] L. Vaidman, Phys. Rev. A 49, 1473 (1994). 